\title{
Das Muskelsystem steuert die Skelettentwicklung
}

\author{
Eckhard Schoenau \\ Universitäts-Kinderklinik Köln, Köln, Deutschland
}

\author{
Schlüsselwörter \\ Knochenfestigkeit • Muskelkraft • Osteoporoseprävention • \\ Sarkopenie
}

\section{Zusammenfassung}

Dieser Übersichtsartikel befasst sich schwerpunktmässig mit den Ansätzen zur Bewertung der Skelettmuskelfunktion und Knochenadaption. Inzwischen geht man davon aus, dass die Knochenfestigkeit und nicht die Knochenmasse das entscheidende Merkmal ist und dass die Knochenfestigkeit in erster Linie von den mechanischen Belastungen des Knochens abhängt. Die maximalen Kräfte, die auf den Knochen einwirken, entstehen durch Muskelkontraktion, so dass sich die Knochenstabilität an die Muskelstärke anpassen muss (die funktionelle Muskel-Knochen-Einheit). Die hier beschriebenen Vorschläge und Empfehlungen umreissen einen ganz neuen Ansatz, der davon ausgeht, dass man die Knochenmasse und Knochenfestigkeit nicht als eine Funktion des Alters sehen sollte. Inzwischen spricht immer mehr dafür, dass die Knochenmasse und -festigkeit eher mit der Muskelfunktion in Beziehung stehen. Geht man von dieser Analyse aus, dann gibt es die sogenannte «Peak Bone Mass», d.h. die maximal zu erreichende Knochenmasse, nicht. Viele der aktuell durchgeführten Studien untersuchen, ob diese neuen Ansätze die Sensitivität und Spezifizität individueller Frakturvorhersagen steigern. Ferner verlagert sich derzeit das Interesse vieler Knochenforscher weg von der Knochenmasse und hin zur Knochengeometrie oder Knochenfestigkeit und deren Beziehung zu dem steuernden Muskelsystem.

Copyright $\odot 2006$ Nestec Ltd., Vevey/S. Karger AG, Basel (c) 2006 Nestec Ltd., Vevey/S. Karger AG, Basel 0250-9652/06/0642-0055\$23.50/0

Fax +41 613061234

E-Mail karger@karger.ch

www.karger.com
Online-Zugriff auf:

www.karger.com/and

\section{Einleitung}

Da die Prävalenz der medizinischen Probleme, die präventive und kurative Ansätze erfordern, einem ständigen Wandel unterworfen ist, stellt jede Generation das Gesundheitssystem vor neue Herausforderungen. In den westlichen Industrienationen werden viele der früher alltäglichen motorischen Aktivitäten inzwischen von den technischen Hilfsmitteln ausgeführt, die uns unsere mechanisierte und computerisierte Welt bietet. Da sich der Bewegungsapparat, d.h. das muskulo-skelettale System, an die biomechanischen Aufgaben und Umweltbedingungen anpasst, hat sich die Körperzusammensetzung eines durchschnittlichen Kindes oder Heranwachsenden in den Industrienationen dramatisch verändert $[1,2]$. Insulinresistenz, Adipositas, Osteopenie und Sarkopenie zählen inzwischen zu den typischen Herausforderungen, denen sich die mit der Gesundheitsprävention und Gesundheitsaufklärung befassten Ärzte aktuell stellen müssen. Noch bis vor einigen Jahrzehnten konzentrierte sich die Pädiatrie in den Industrieländern auf die Prävention der Rachitis und der durch zu geringe Kalorienzufuhr hervorgerufenen Mangelernährung. Heute stellen die durch mangelnde Bewegung hervorgerufene Atrophie der Muskeln und Knochen und die in Verbindung mit zu hoher Energiezufuhr stehende Überernährung die grössten Probleme dar. Ferner wurden, aufgrund der besseren medizinischen Versorgung chronisch kranker Kinder, die sekundären Erkrankungen des muskulo-skelettalen Systems zu einem wichtigen Problem. Das zur Bewertung der Interaktionen zwischen Muskeln und Skelett 
Abb. 1. Ein auf der Mechanostat-Theorie basierendes funktionelles Modell der Knochenentwicklung. Kernstück der Regulierung der Knochenentwicklung ist der Regelkreis zwischen Knochenverformung (Gewebeverformung) und Knochenfestigkeit. Während des Wachstums muss sich dieses homöostatischeSystem fortwährend an die externen Herausforderungen anpassen. Die aufgeführten Faktoren modulieren diverse Aspekte dieses zentralen Regelkreises. Mit freundlicher Genehmigung aus Rauch und Schoenau [29].

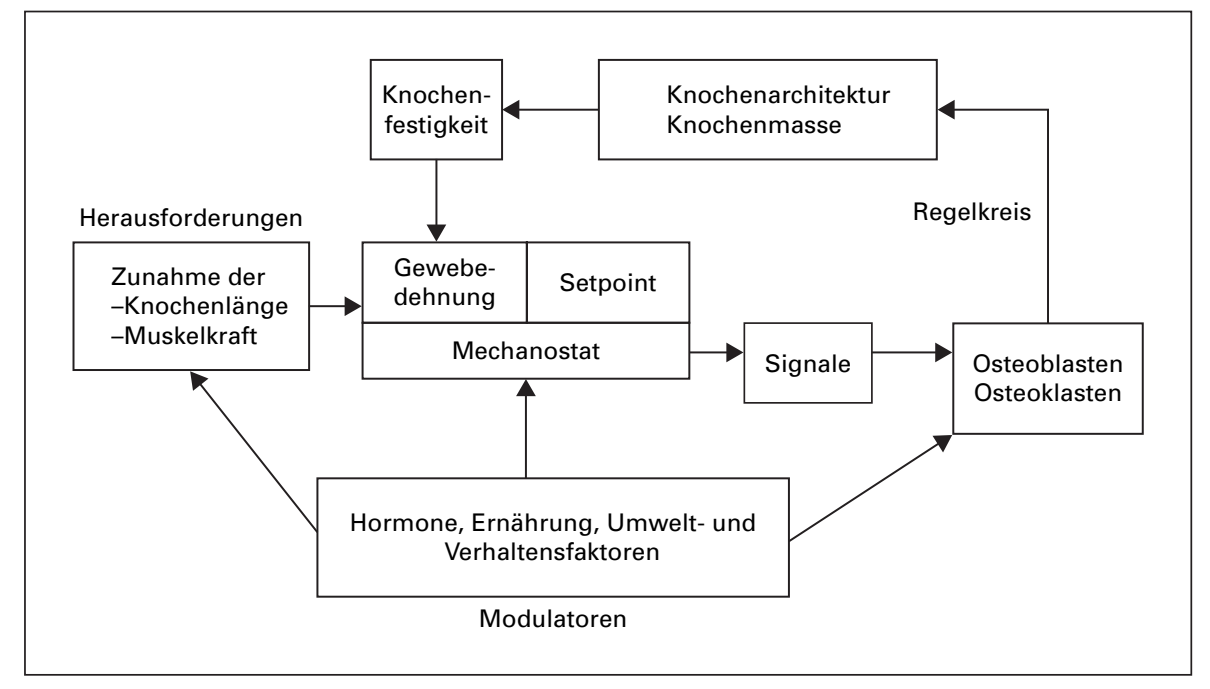

erforderliche Wissen und Know-how wird somit in der Pädiatrie an Bedeutung gewinnen. Einblicke in die Entwicklung des muskulo-skelettalen Systems und die Beurteilung dieses Vorgangs werden für alle Personen, die mit der Verbesserung der medizinischen Versorgung in unseren Gesellschaften betraut sind, an Bedeutung gewinnen. Dieser Übersichtsartikel beschäftigt sich mit den mechanischen Einflüssen der Skelettentwicklung.

\section{Die Mechanostat-Hypothese von Harold Frost}

Mit Mechanostat bezeichnet man die Kombination von Faktoren, die zu gesunden und belastbaren Knochen führt, die den Bedürfnissen von Amphibien, Vögeln, Säugetieren und Reptilien aller Grössen, Alter und Geschlecht gerecht werden. Das Modell verbindet die Mechanismen des Knochenaufbaus (Modelling) und -umbaus (Remodelling), die für diese Mechanismen geltenden Schwellen, die Mediatoren-Mechanismen im Knochenmark und die Signalgebungsmechanismen, die diese und möglicherweise andere Mechanismen verbinden. Aus mechanischen Gründen bestimmt der sich hieraus ergebende negative Regelkreis, ob, wann und wo Knochen eine höhere Festigkeit benötigen oder ob kein Knochenaufbau nötig ist. Diverse nicht mechanische Einflüsse, u.a. Hormone und andere humorale Mechanismen, können die Auswirkungen des Mechanostats auf die Knochenfestigkeit verändern (unterstützen oder behindern). Man könnte den Mechanostat mit der in einem Auto vorliegenden Kombination aus Lenkung, Bremsen und Gaspedal vergleichen. Bedient man sich dieser Ana- logie, so entsprächen die Osteoblasten und Osteoklasten den Rädern des Fahrzeugs und die mechanische Belastung dem Fahrer (Abb. 1) [3-5].

Während der Wachstumsphase wird der Knochenund Muskelaufbau von Kräften bestimmt, die mit der Schwerkraft und der Bewegung zusammenhängen $[4,6$, 7]. Am stärksten wirken sich die Muskelkräfte auf die Knochen aus. Werden die Muskeln und Knochen während des Wachstums nicht ausreichend belastet, so bildet sich ein Muskel, dem die funktionelle Kapazität fehlt, und ein Knochen, der nicht die für seine Funktion einzigartige Form aufweist [8]. Die intrinsische Beziehung zwischen Muskeln und Knochen wird in der Mechanostat-Theorie beschrieben, die besagt, dass sich eine Steigerung der maximalen Muskelkraft, wie sie während des Wachstums oder infolge erhöhter Belastung auftritt, auf die Masse, Grösse und Festigkeit der Knochen auswirkt. Entlastung (Inaktivität oder Immobilisierung) dagegen reduziert die Muskelentwicklung (und damit Muskelkraft) und wirkt sich somit negativ auf die Knochenmasse, -grösse und -festigkeit aus.

$\mathrm{Zu}$ den Faktoren, die für eine ordnungsgemässe Funktion des Mechanostats massgeblich sind, zählen der normale Status aller am Mechanostat beteiligter Zellen (Osteozyten, -blasten und -klasten), eine normale mechanische Belastung des Skeletts und die endokrine Stoffwechselumgebung [9]. Die Feineinstellung des Mechanostats erfolgt über physiologische Setpoints, die als Schwellen für die Initiierung oder Hemmung des Knochen-Modelling und -Remodelling dienen. Die Setpoints des Mechanostats sind genetisch festgelegt, werden aber über die Hormone gesteuert. So wird beispielsweise ver- 


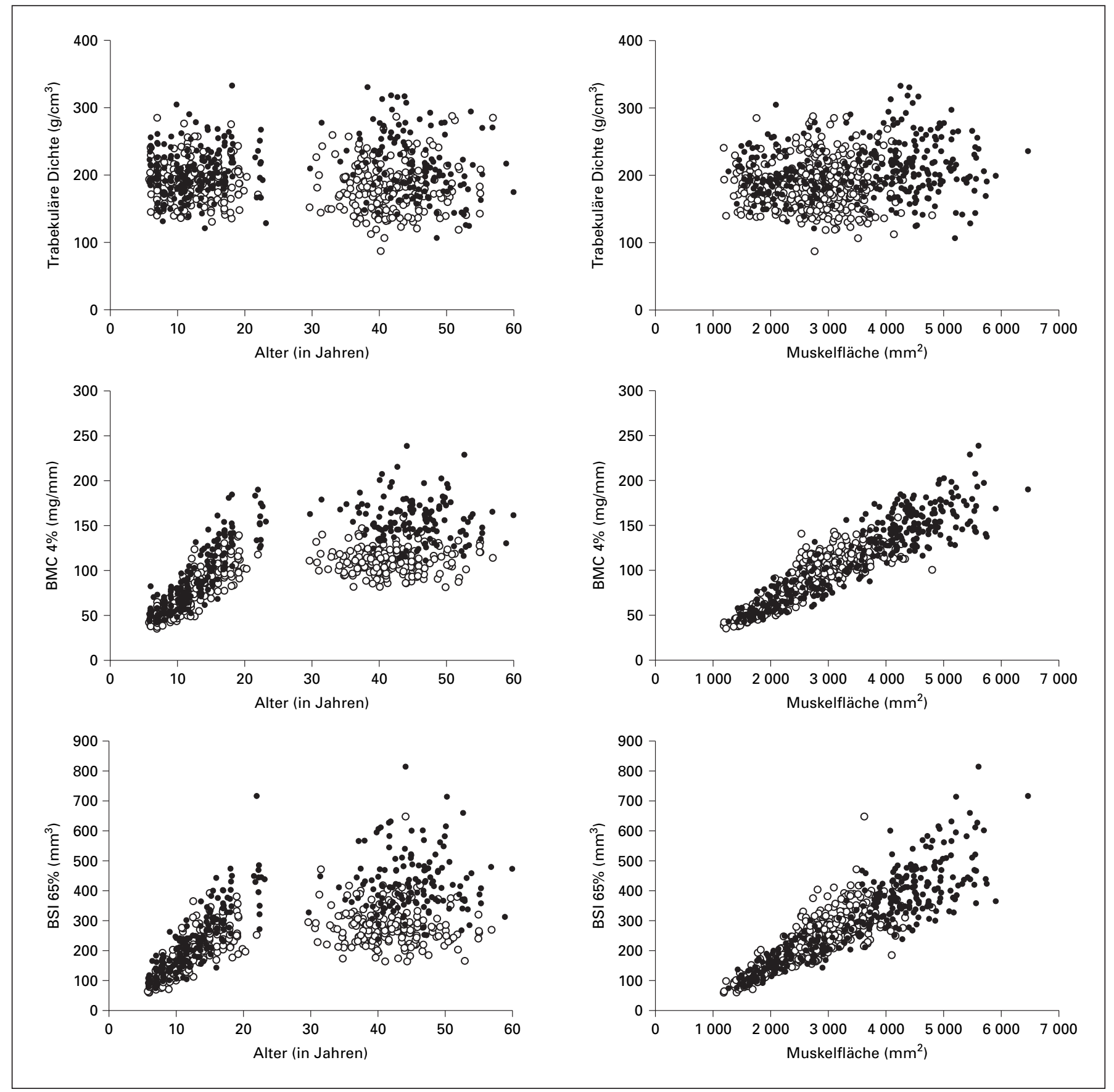

Abb. 2. Trabekuläre Dichte, Knochenmineralgehalt (BMC 4\%) und Knochenfestigkeitsindex (BSI 65\%) im Verhältnis zu Alter und Muskelfläche. $\bigcirc$ = Frauen; $=$ Männer. Mit freundlicher Genehmigung aus Schoenau [13].

mutet, die Schwellenwerte für die Initiierung des Knochen-Modellings und -Remodellings seien bei niedrigen Östrogenkonzentrationen höher. Die Hormone wirken sich auf die Empfindlichkeit des Mechanostats aus, d.h. die Empfindlichkeit, mit der ein Knochen seine Masse, seine Geometrie bzw. seine strukturellen Eigenschaften an die durch Belastung hervorgerufenen Knochenverformungen anpasst [9]. 


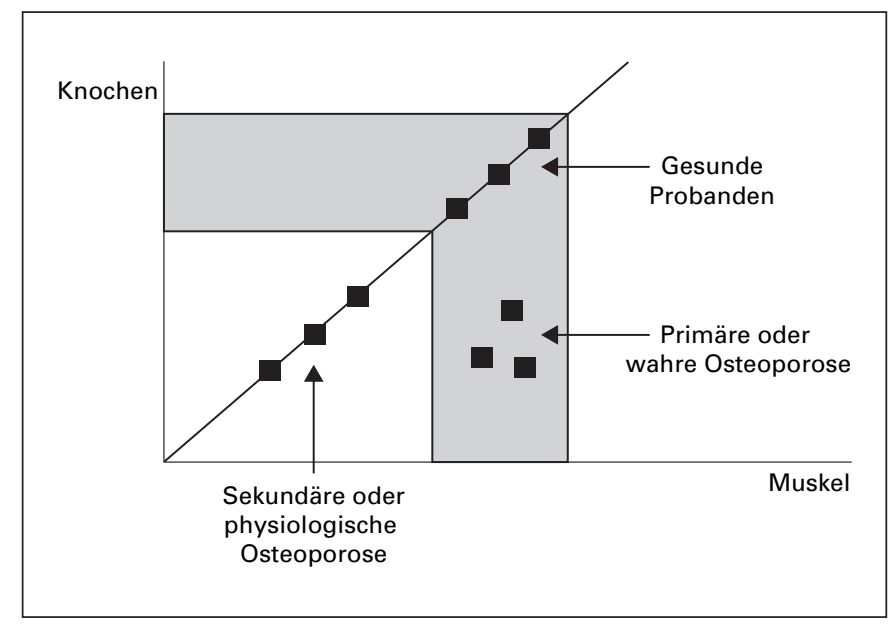

Abb. 3. Die «funktionelle Muskel-Knochen-Einheit». Primäre Osteoporose: Keine Anpassung der Knochenstruktur bzw. -masse an die Muskelentwicklung. Sekundäre Osteoporose: Gestörte Muskelentwicklung, aber normal adaptiertes Skelett. Mit freundlicher Genehmigung aus Schoenau [13].

Bewegung und Ernährung sind die zwei wichtigsten Umweltfaktoren, von denen man weiss, dass sie sich auf die Muskel- und Knochenentwicklung auswirken. Bewegung wirkt direkt über die Muskelwirkung und indirekt über die hormonelle Steuerung; man geht davon aus, dass sich Bewegung während des Wachstums auf das Knochen-Modelling und damit auf die Knochengeometrie auswirkt.

\section{Die Entwicklung der Knochendichte, -masse und -festigkeit}

Abbildung 2 zeigt das Verhältnis von Alter und Muskelfläche zu trabekulärer Dichte, Knochenmasse (Knochenmineralgehalt, BMC) und Knochenfestigkeitsindex (BSI) bei einer gesunden Referenzpopulation. Über die bei diesen Personen mit Hilfe der peripheren quantitativen Computertomographie erhobenen anthropometrischen Daten und Ergebnisse wurde bereits in einer früheren Veröffentlichung berichtet $[10,12]$. Die trabekuläre Dichte als Index der Gewebedichte hängt weder vom Alter noch von der Muskelentwicklung ab. Bei Knochenmineralgehalt und Knochenfestigkeitsindex scheint lediglich in der Kindheit eine Altersabhängigkeit vorzuliegen. Im Gegensatz dazu ist jedoch sowohl beim Knochenmineralgehalt als auch beim Knochenfestigkeitsindex in der Kindheit wie im Erwachsenenalter eine starke lineare
Korrelation mit der Muskelentwicklung festzustellen. Laut diesen Daten handelt es sich bei der Knochendichte mehr oder minder um eine «Konstante», während der Knochenmineralgehalt und der Knochenfestigkeitsindex Funktionen der Muskelentwicklung sind. Ausgehend von diesen Überlegungen empfahlen wir vor einigen Jahren, die analysierten Knochendaten mit Indikatoren der Muskelentwicklung in Verbindung zu setzen. Die Analyse der sogenannten «Muskel-Knochen-Einheit» anstelle von altersabhängigen Referenzdaten (Abb. 3) sollte zu einem besserem Verständnis der Physiologie und Pathophysiologie der Knochenentwicklung beitragen [13].

\section{Einfluss der Pubertät auf die «Muskel-Knochen- Einheit»}

Im Jahr 1995 warf Frost (persönlicher Kommentar) die wichtige Frage auf, ob das Hormon Östrogen dazu führen könnte, dass Mädchen während der Wachstumsphase mehr Knochenmasse als eigentlich zur Bewegung nötig aufbauen, um so Kalziumreserven für eine zukünftige Schwangerschaft und Laktation anzulegen. Die von Zanchetta et al. [14] ermittelten Daten stützen diese Hypothese. Mit Hilfe der dualen Röntgenabsorptiometrie schätzte diese Gruppe den Gesamtkörper-BMC und die Muskelmasse bei 778 gesunden argentinischen Kindern weisser Hautfarbe. Schiessl et al. [15] unterzogen diese Daten einer erneuten Analyse und fanden heraus, dass bei Mädchen in der Pubertät ein stärkerer Zuwachs an Knochenmasse zu verzeichnen ist als bei Jungen mit einer vergleichbaren Muskelmasse. Da bei Verwendung des Gesamtkörper-BMC und der Muskelmasse als Indikatoren für Knochenfestigkeit und Muskelkraft methodologische und analytische Unsicherheiten bestehen und diese Erkenntnisse möglicherweise äusserst wichtig waren, schienen weitere Studien zur Bestätigung der Ergebnisse nötig. Bei gesunden Kindern und Jugendlichen im Alter zwischen 6 und 22 Jahren und bei Erwachsenen, die an der DONALD-Studie (Dortmund Nutritional and Anthropometric Longitudinally Designed Study) teilnahmen, wurde mit Hilfe der peripheren quantitativen Computertomographie die kortikale Fläche des Radius als Indikator für die Knochenfestigkeit und die Muskelfläche als Indikator für die Muskelkraft berechnet [16, 17].

Bei allen Kindern, Jugendlichen und Erwachsenen bestand eine starke Korrelation zwischen der Muskelfläche und der kortikalen Fläche des Radius. Abbildung 4 zeigt die Korrelationen zwischen der Muskelfläche und der 


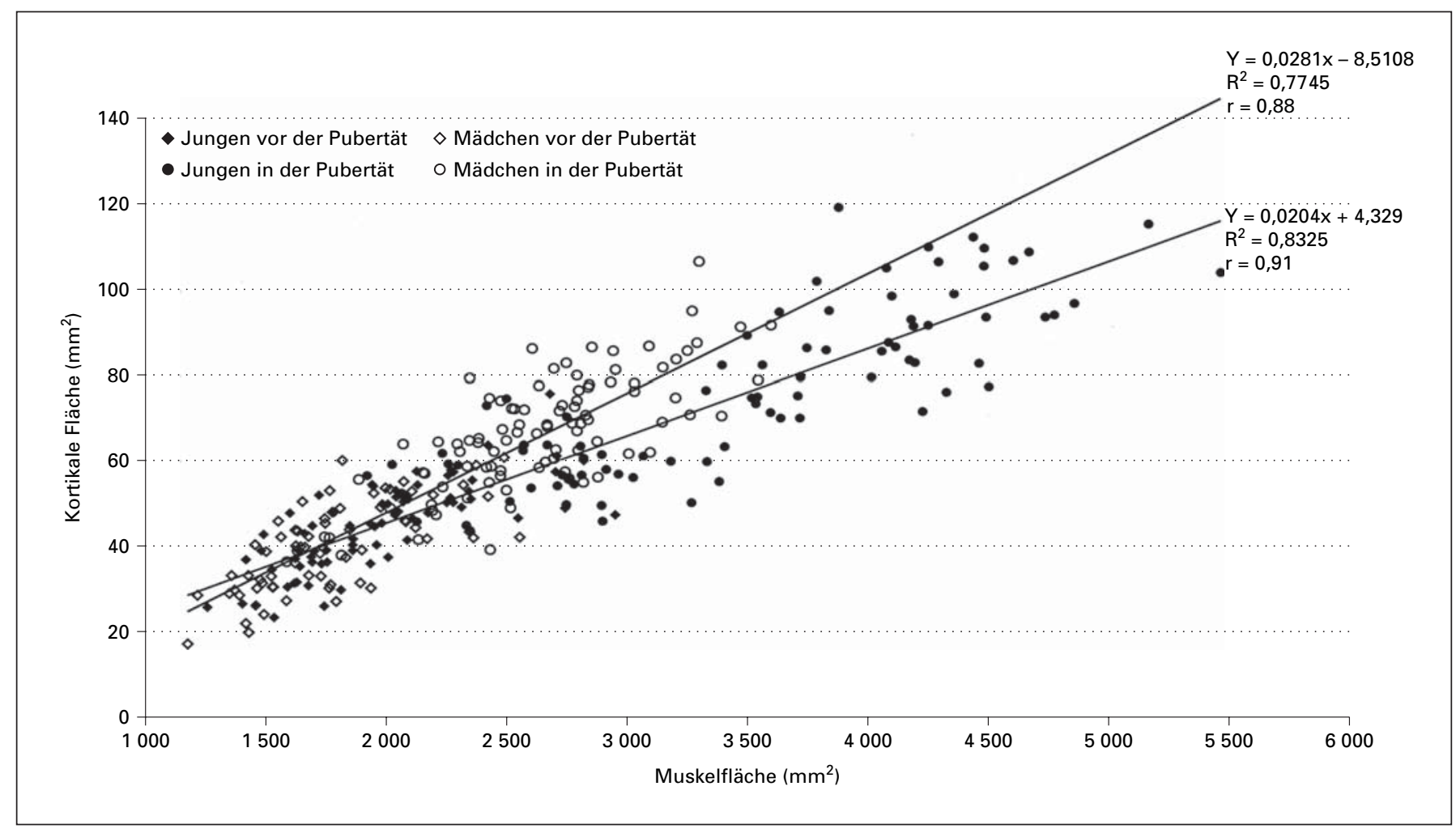

Abb. 4. Kortikale Knochenfläche im Verhältnis zur Muskelfläche bei Jungen und Mädchen vor und während der Pubertät. Mit freundlicher Genehmigung aus Schoenau et al. [16]; Copyright 2000, The Endocrine Society.

kortikalen Fläche bei männlichem und weiblichem Geschlecht. Vor der Pubertät war das Verhältnis von Muskelfläche zu kortikaler Fläche bei Jungen und Mädchen gleich. Nach der Pubertät jedoch war die kortikale Fläche im Verhältnis zur Muskelfläche bei Mädchen grösser als bei Jungen. Im Pubertätsstadium 3 wurde bei Mädchen eine im Verhältnis zur Muskelfläche grössere kortikale Fläche nachgewiesen. Abbildung 5 zeigt das Verhältnis zwischen periostalem bzw. endostalem Umfang und Muskelfläche. Bezüglich der Korrelation zwischen Muskelfläche und periostalem Umfang waren bei Männern und Frauen keine signifikanten Unterschiede festzustellen. Im Gegensatz dazu war das Verhältnis von Muskelfläche zu endostalem Umfang zwischen den Geschlechtern signifikant unterschiedlich. Diese Daten untermauern die Thesen von Frost und dem sogenannten Utah-Paradigma (Utah Paradigm of Skeletal Physiology) zur Knochenentwicklung in der Kindheit und Jugend. Die Maximalkräfte, die auf die Knochen einwirken, gehen von den Muskeln aus. Um die Knochenfestigkeit und -masse an diese Belastungen anzupassen, legen bestimm-

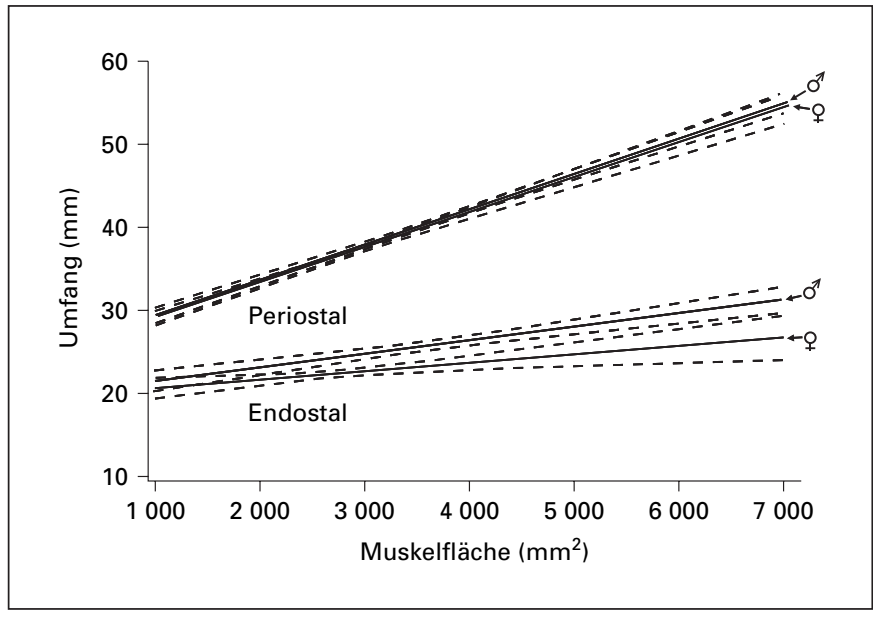

Abb. 5. Regressionslinien und 95\%-Konfidenzintervalle der Korrelation zwischen Muskelfläche und periostalem bzw. endostalem Umfang. Mit freundlicher Genehmigung aus Schoenau et al. [16]; Copyright 2000, The Endocrine Society. 


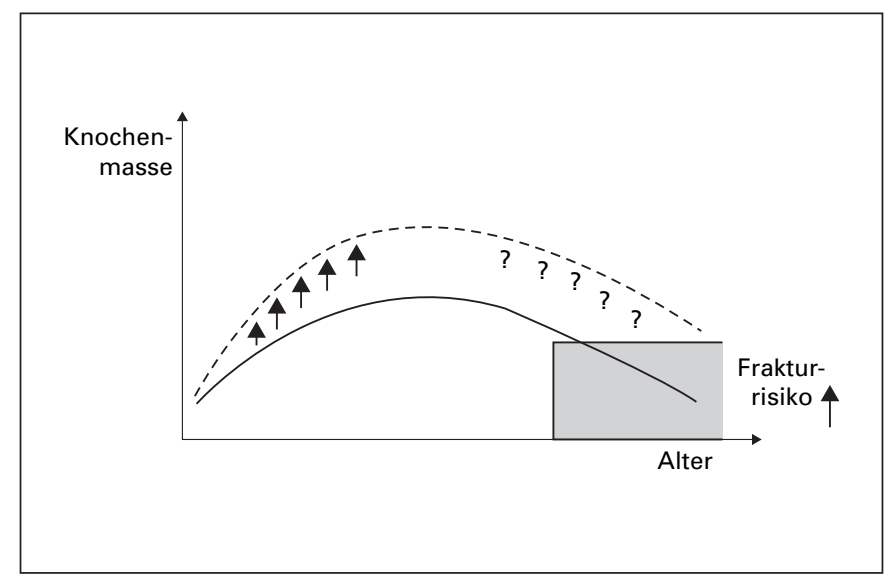

Abb. 6. Konzept der Peak Bone Mass (maximalen Knochenmasse): Frakturprävention durch Verbesserung der individuellen Knochenmasse im Kindes- und Jugendalter? Mit freundlicher Genehmigung aus Springer Science und Business Media [Pediatr Nephrol 2004;19:825-831].

te Schwellenbereiche der Knochenbelastung fest, an welcher Stelle durch das Modelling ein Knochenzuwachs und eine Stärkung der Knochen erfolgt und wann Knochen mittels Remodelling konserviert oder abgebaut werden, ähnlich wie bei einem Thermostat, bei dem die unterschiedlichen Einstellungen die Heiz- und Kühlsysteme in einem Haus steuern. Falls sich die Östrogene auf die Sensitivität des Mechanostats auswirken und bei Mädchen in der Pubertät die Schwellenwerte für das Remodelling herabsetzen, müsste die Knochenmasse bei Mädchen aufgrund des geringeren, von den Remodelling-Prozessen abhängigen Knochenabbaus schneller ansteigen als bei Jungen mit einer vergleichbaren Muskelstärke. Die hier aufgeführten Ergebnisse ergänzen die von Zanchetta et al. [14], Schiessl et al. [15] und Ferretti et al. [18] durchgeführten Studien und untermauern die angeführte These.

\section{Wirkt sich eine optimale Peak Bone Mass im Kindes- und Jugendalter langfristig aus?}

Ausgehend von der allgemein gültigen These eines optimalen Knochenmasseaufbaus untersuchten viele Studien die Auswirkungen der Kalziumaufnahme auf die Entwicklung der Knochenmasse (Abb. 6). Eine Zwillingsstudie ergab, dass eine höhere Kalziumzufuhr sich bei Kindern geringfügig positiv auf die Knochenmasse auswirkt [19]. Die Kalziumaufnahme in der Pubertät

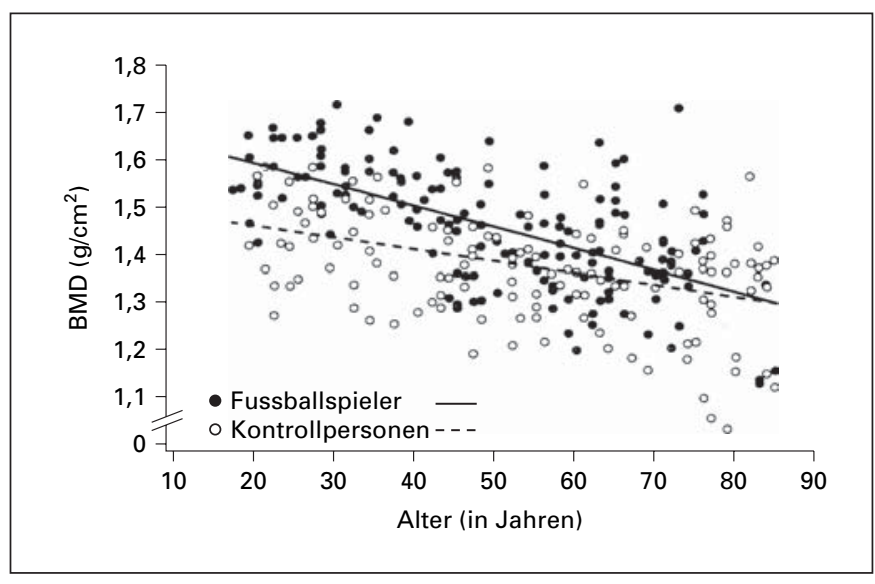

Abb. 7. Knochenmineraldichte (BMD) der Beinknochen aktiver und ehemaliger Fussballspieler verglichen mit Kontrollpersonen. Mit freundlicher Genehmigung aus Johnston et al. [19].

hatte interessanterweise keinen Einfluss auf die Knochenmasse, obwohl der Kalziumzufuhr während des pubertären Wachstumsschubs allgemein grosse Bedeutung zugemessen wird. Eine Follow-up-Untersuchung der Zwillinge ergab, dass sich die signifikanten Unterschiede in der Knochenmineraldichte zwischen den Zwillingen mit und den Zwillingen ohne Kalziumsupplementation nach Absetzen der Kalziumtabletten nivellierten.

In einer weiteren Studie führte eine kontrollierte, über 18 Monate durchgeführte Supplementierung mit Kalzium zu einer höheren Knochenmineralmasse, wobei sich die Zunahmen innerhalb von 18 Monaten nach der Studie wieder zurückbildeten [20]. Studien zu biochemischen Markern, die das Modelling und Remodelling der Knochen widerspiegeln, ergaben, dass die Kalziumaufnahme zwar die Umbaurate (Remodelling Rate) senkte, sich aber nicht auf das Modelling auswirkte [21].

Diese Daten decken sich mit der Hypothese, laut derer eine Kalziumsupplementation die Umbauaktivität des Skeletts senkt. Die niedrigeren Umbauraten sind möglicherweise auf die gesenkten PTH (Parathormon)-Spiegel zurückzuführen und würden dann die kortikale Porosität reduzieren. Dieser offensichtliche Zuwachs an Knochenmasse ist jedoch vollständig reversibel, sobald die Umbauraten wieder steigen. Wichtig ist, dass die Kalziumsupplementation sich nicht stimulierend auf das Modelling auszuwirken scheint, das im Kindes- und Jugendalter der wichtigste Vorgang zur Erhöhung der Knochenstärke ist (kortikale Dicke und kortikale Fläche). 
Auch die langfristigen Auswirkungen der Bewegung sind möglicherweise reversibel. Eine an Fussballspielern durchgeführte Studie ergab, dass Bewegung in der Jugend hinsichtlich der maximalen Knochenmineraldichte $\mathrm{zu}$ einer hohen und klinisch relevanten Nutzwirkung führt, dass aber, sobald der Sport eingestellt wird, ein beschleunigter Abbau der Knochenmineraldichte eintritt (Abb. 7) [22]. Somit war die verbleibende Nutzwirkung hinsichtlich der Knochenmineraldichte bei ehemaligen Fussballspielern, die 60 Jahre und älter waren und schon seit über 35 Jahren nicht mehr Fussball spielten, nicht mehr signifikant. Auch die Frakturrate war altersgemäss und nicht etwa niedriger. Pajamaki et al. [23] berichteten aus einem Rattenversuch ähnliche Ergebnisse. Eine durch Bewegung hervorgerufene Nutzwirkung für die Knochen geht verloren, sobald kein Sport mehr ausgeübt wird, und deshalb bedarf es wahrscheinlich eines kontinuierlichen Trainings, um die im Jugendalter durch den Sport erzielten positiven Wirkungen auch im Erwachsenenalter aufrechtzuerhalten.

Andererseits haben Tierversuche ergeben, dass eine vorübergehende, in einem frühen Lebensabschnitt stattfindende Störung der Zunahme der Knochenmasse sich eventuell überhaupt nicht auf die maximale Knochenmasse (Peak Bone Mass) auswirkt [24]. Die Studie belegte, dass manche Bereiche des jugendlichen Skeletts nicht vollständig umgebaut, sondern tatsächlich als Ganzes durch das Skelettwachstum ersetzt werden. Wächst ein Knochen in der Jugend und weitet sich dabei die Markhöhle, so wird ein in einem früheren Lebensabschnitt gebildeter Knochen unabhängig von seiner Qualität nach und nach resorbiert und durch einen neuen Knochen ersetzt. Dank diesem Mechanismus wird eine unzureichende Knochenentwicklung sozusagen «repariert», sobald keine negativen Einflüsse mehr vorliegen.

Zusammenfassend ist zu sagen, dass kurzfristige Auswirkungen auf die Knochenmasse, gleichgültig ob diese positiver oder negativer Natur sind, keine langfristigen Folgen zu haben scheinen. Das Skelettsystem scheint sich eher an die aktuellen Anforderungen als an die Anforderungen einer früheren Entwicklungsphase anzupassen.

\section{Ausblick und neue Konzepte}

Die folgenden Vorschläge und Empfehlungen umreissen ein neues Konzept, das auf den oben dargelegten Überlegungen basiert, für das bisher jedoch noch nicht belegt wurde, dass es im Einzelnen korrekt ist. Man nimmt an, dass die Knochenmasse nicht mit dem Alter zusammenhängt. Inzwischen sprechen immer mehr Anzeichen dafür, dass ein Zusammenhang zwischen Knochenmasse und Knochengrösse [25] bzw. Muskelfunktion [25-31] besteht.

Geht man von dieser Darlegung aus, dann gibt es die maximale Knochenmasse, die sogenannte «Peak Bone Mass», nicht. Derzeit werden viele Studien durchgeführt, um zu bewerten, ob diese Ansätze die Sensitivität und Spezifizität der individuellen Frakturvorhersage erhöhen.

Ferner verlagert sich das Interesse vieler Knochenforscher weg von der Knochenmasse und hin zur Knochengeometrie oder Knochenfestigkeit [32, 33]. Die Knochenmasse wird als Indikator der Knochenfestigkeit betrachtet, es können aber auch gängige Verfahren, wie die duale Röntgenabsorptiometrie, die Radiogrammetrie und die Computertomographie, eingesetzt werden, um die Variablen der Knochengeometrie, wie z.B. kortikale Dicke, kortikale Fläche und Trägheitsmoment, zu messen. Zukünftige Studien werden zeigen, ob die kombinierte Analyse der Knochengeometrie und der Knochenmasse das Konzept der Peak Bone Mass verfeinert oder dazu führt, dass dieses Konzept verworfen wird.

Die Analyse der Knochenmasse erweist sich derzeit für epidemiologische Studien zu Faktoren, die sich auf die Knochenentwicklung auswirken können, als äusserst nützlich. Die Knochenmasse darf jedoch nicht als äusserst sensitives und spezifisches Mass für das individuelle Frakturrisiko angesehen werden. Das Konzept der Peak Bone Mass geht davon aus, dass Frakturen im Erwachsenenalter durch eine optimale Knochenentwicklung im Kindes- und Jugendalter vermieden werden können. Inzwischen ist jedoch klar, dass starke Knochen im Jugendalter nicht automatisch das Auftreten von Frakturen im Alter verhindern. Es könnte jedoch möglich sein, dass starke Knochen auch stark bleiben, wenn die betreffende Person auch weiterhin den gesunden Lebensstil pflegt, der die Knochen überhaupt erst stark gemacht hat. $\mathrm{Ob}$ diese Hypothese korrekt ist, bleibt jedoch abzuwarten. 


\section{Literatur}

$>1$ Strock GA, Cottrell ER, Abang AE, et al: Childhood obesity: a simple equation with complex variables. J Long Term Eff Med Implants 2005;15:15-32.

$\checkmark 2$ Popkin BM: The nutrition transition: an overview of world patterns of change. Nutr Rev 2004;62:S140-S143.

$\checkmark 3$ Frost HM: Bone 'mass' and the 'mechanostat': a proposal. Anat Rec 1987;219:1-9.

$\checkmark 4$ Burr DB: Muscle strength, bone mass, and age-related bone loss. J Bone Miner Res 1997;12:1547-1551.

$\checkmark 5$ Schoenau E, Frost HM: The 'muscle-bone unit' in children and adolescents. Calcif Tissue Int 2002;70:405-407.

$>6$ Turner CH: Muscle-bone interactions, revisited. Bone 2000;27:339-340.

$>7$ Frost HM: On our age-related bone loss: insights from a new paradigm. J Bone Miner Res 1997;12:1539-1546.

$>8$ Frost HM, Schonau E: The 'muscle-bone unit' in children and adolescents: a 2000 overview. J Pediatr Endocrinol Metab 2000; 13:571-590.

$>9$ Cointry GR, Capozza RF, Negri AL, et al: Biomechanical background for a noninvasive assessment of bone strength and musclebone interactions. J Musculoskelet Neuronal Interact 2004;4:1-11.

10 Neu CM, Manz F, Rauch F, et al: Bone densities and bone size at the distal radius in healthy children and adolescents: a study using peripheral quantitative computed tomography. Bone 2001;28:227-232.

$\checkmark 11$ Neu CM, Rauch F, Manz F, Schoenau E: Modeling of cross-sectional bone size, mass and geometry at the proximal radius: a study of normal bone development using peripheral quantitative computed tomography. Osteoporos Int 2001;12:538-547.

12 Schoenau E, Neu CM, Rauch F, Manz F: The development of bone strength at the proximal radius during childhood and adolescence. J Clin Endocrinol Metab 2001;86: 613-618.
13 Schoenau E: From mechanostat theory to de velopment of the 'Functional Muscle-BoneUnit'. J Musculoskelet Neuronal Interact 2005;5:232-238.

14 Zanchetta JR, Plotkin H, Alvarez Filgueira ML: Bone mass in children: normative values for the 2-20-year-old population. Bone 1995;16(suppl):393S-399S.

15 Schiessl H, Frost HM, Jee WS: Estrogen and bone-muscle strength and mass relationships. Bone 1998;22:1-6.

16 Schoenau E, Neu CM, Mokov E, et al: Influence of puberty on muscle area and cortical bone area of the forearm in boys and girls. J Clin Endocrinol Metab 2000;85:1095-1098.

17 Kersting M, Sichert-Hellert W, Lausen B, et al: Energy intake of 1 to 18 year old German children and adolescents. Z Ernahrungswiss 1998;37:47-55.

18 Ferretti JL, Capozza RF, Cointry GR, et al: Gender-related differences in the relationship between densitometric values of wholebody bone mineral content and lean body mass in humans between 2 and 87 years of age. Bone 1998;22:683-690

19 Johnston CC Jr, Miller JZ, Slemenda CW, et al: Calcium supplementation and increases in bone mineral density in children. N Engl J Med 1992;327:82-87.

20 Lee WT, Leung SS, Leung DM, Cheng JC: A follow-up study on the effects of calciumsupplement withdrawal and puberty on bone acquisition of children. Am J Clin Nutr 1996; 64:71-77.

21 Slemenda CW, Peacock M, Hui S, et al: Reduced rates of skeletal remodeling are associated with increased bone mineral density during the development of peak skeletal mass. J Bone Miner Res 1997;12:676-682.

22 Karlsson MK, Linden C, Karlsson C, et al: Exercise during growth and bone mineral density and fractures in old age. Lancet 2000;355:469-470.
23 Pajamaki I, Kannus P, Vuohelainen T, et al: The bone gain induced by exercise in puberty is not preserved through a virtually lifelong deconditioning: a randomized controlled experimental study in male rats. J Bone Miner Res 2003;18:544-552.

24 Gafni RI, McCarthy EF, Hatcher T, et al: Recovery from osteoporosis through skeletal growth: early bone mass acquisition has little effect on adult bone density. FASEB J 2002 16:736-738.

25 Kroger H, Vainio P, Nieminen J, Kotaniemi A: Comparison of different models for interpreting bone mineral density measurements using DXA and MRI technology. Bone 1995; 17:157-159.

26 Molgaard C, Thomsen BL, Michaelsen KF: Influence of weight, age and puberty on bone size and bone mineral content in healthy children and adolescents. Acta Paediatr 1998;87:494-499.

27 Lu PW, Cowell CT, Lloyd-Jones SA, et al: Volumetric bone mineral density in normal subjects, aged 5-27 years. J Clin Endocrinol Metab 1996;81:1586-1590.

28 Schoenau E, Neu CM, Beck B, et al: Bone mineral content per muscle cross-sectional area as an index of the functional musclebone unit. J Bone Miner Res 2002;17:10951101.

29 Rauch F, Schoenau E: The developing bone: slave or master of its cells and molecules? Pediatr Res 2001;50:309-314.

-30 Finkelstein JS, Neer RM, Biller BM, et al: Osteopenia in men with a history of delayed puberty. N Engl J Med 1992;326:600-604.

31 Hogler W, Briody J, Woodhead HJ, et al: Importance of lean mass in the interpretation of total body densitometry in children and adolescents. J Pediatr 2003;143:81-88.

32 Seeman E: From density to structure: growing up and growing old on the surfaces of bone. J Bone Miner Res 1997;12:509-521.

$\checkmark 33$ Seeman E: Periosteal bone formation - a neglected determinant of bone strength. N Engl J Med 2003;349:320-323. 